\title{
Ultrasonic assessment of osseointegration phenomena at the bone-implant interface using convolutional neural network
}

\author{
Yunsang Kwak ${ }^{1,2}$, Vu-Hieu Nguyen ${ }^{3,4}$, Yoann Hériveaux ${ }^{1}$, Pierre Belanger ${ }^{5}$, Junhong Park $^{2}$, \\ 5 Guillaume Haïat ${ }^{1, *}$ \\ ${ }^{1}$ CNRS, MSME, UPEC, UGE, F-94010 Creteil, France \\ ${ }^{2}$ Department of Mechanical Engineering, Hanyang University, 222 Wangsimni-ro, Seongdong- \\ gu, 04763 Seoul, Korea \\ $10 \quad{ }^{3}$ Univ Paris Est Creteil, CNRS, MSME, F-94010 Creteil, France \\ ${ }^{4}$ Univ Gustave Eiffel, MSME, F-77454 Marne-la-Vallée, France \\ ${ }^{5}$ Department of Mechanical Engineering, École de Technologie Supérieure, 1100 Rue Notre- \\ Dame O, Montreal, Quebec, H3C1K3, Canada. \\ * Corresponding author: guillaume.haiat@ cnrs.fr (G.H.)

\begin{abstract}
Although endosseous implants are widely used in the clinic, failures still occur and their clinical performance depends on the quality of osseointegration phenomena at the bone-implant interface (BII), which are given by bone ingrowth around the BII. The difficulties to ensure clinical

20 reliability come from the complex nature of this interphase related to the implant surface roughness and the presence of a soft tissue layer (non-mineralized bone tissue) at the BII. The aim of the present study is to develop a method to assess the soft tissue thickness at the BII based on the analysis of its ultrasonic response using simulation based-convolution neural network (CNN). A large-annotated dataset was constructed using a 2-D finite element model in the frequency domain 25 considering a sinusoidal description of the BII. The proposed network was trained by the synthesized ultrasound responses and was validated by a separate dataset from the training process. The linear correlation between actual and estimated soft tissue thickness shows excellent $R^{2}$ values equal to $99.52 \%$ and $99.65 \%$, and narrow limit of agreement corresponded to [-2.56, $4.32 \mu \mathrm{m}]$ and $[-15.75,30.35 \mu \mathrm{m}]$ for the microscopic and macroscopic roughness respectively, supporting the reliability of the proposed assessment for the osseointegration phenomena.
\end{abstract}

Keywords: Bone-implant interface, osseointegration, quantitative ultrasound, Finite element method, Convolutional neural network.

Running title: Implant ultrasound assessment with neural network.

35 PACS numbers: 43.80.Ev, 43.80.Vj, 43.20.Gp, 43.20.El 


\section{Introduction}

Although implants are now widely used in dental and orthopedic surgery, there remain risks of failure which may have dramatic consequences and are difficult to anticipate. The surgical

40 success depends on the quality of osseointegration phenomena, which corresponds to the formation of bone tissue in contact with the implant (Haiat et al., 2014). During osseointegration phenomena, collagen-rich tissue forms around the implant and is then mineralized, which corresponds to a transformation from soft tissue into a solid material(Moerman et al., 2016). Aseptic loosening, which depends on osseointegration phenomena, is the most common cause of surgical failure of

45 endosseous implant surgery (Pilliar et al., 1986). Therefore, the non-invasive assessment of osseointegration phenomena is important in order to maximize the clinical success of such intervention (Khan et al., 2012). However, it remains difficult to assess the evolution of the implant stability, which is given by the biomechanical properties of the bone-implant interface (BII) (Mathieu et al., 2014).

Different biomechanical techniques have been proposed to investigate the BII properties because techniques using X-rays and Magnetic Resonance Imaging have strong limitations due to the presence of metal (Shalabi et al., 2007; Gill and Shellock, 2012). To investigate the BII properties, impact methods (Van and Wilson, 1991; Schulte and Lukas, 1992; Michel et al., 2016;

55 Goossens et al., 2017; Dubory et al., 2020) and resonance frequency analysis (Meredith et al., 1996; Georgiou and Cunningham, 2001; Pastrav et al., 2009) have been employed but remain limited in terms of resolution and of sensitivity to investigate the biomechanical properties of the BII (Aparicio et al., 2006; Seong et al., 2009). Approaches using quantitative ultrasound (QUS) seem promising to retrieve information on the BII because it is non-invasive, easy to use and non-

60 ionizing. Preliminary studies using coin-shaped implant models have evidenced a significant decrease of the reflection coefficient of the BII as a function of healing time (Mathieu et al., 2012; Fraulob et al., 2020), which can be explained by increases of the bone-implant contact (BIC) ratio, of the bone Young's modulus (Vayron et al., 2012) and of bone mass density (Mathieu et al., 2011; Vayron et al., 2014b) as a function of healing time. The sensitivity of the reflection coefficient of

65 the BII to compressive stresses was also investigated (Hériveaux et al., 2019b). Moreover, in vitro (Vayron et al., 2018b) and in vivo (Vayron et al., 2014c; Vayron et al., 2018a) studies have proven the potentiality of QUS techniques to evaluate dental implant stability and have evidenced a better 
resolution compared to the resonance frequency analysis technique, which is currently employed in the clinics. Despite the interesting performances of QUS techniques to retrieve information on the BII properties, experimental approaches are limited when it comes to understand the determinant of the QUS response of the BII because of the various parameters such as surface roughness, bone quality and bone quantity that are impossible to control and that evolve in parallel.

Acoustical modeling is the only way to gain a further understanding of the interaction 75 between ultrasonic waves and the BII. Therefore, numerical studies considering 2-D and 3-D finite element modeling (Vayron et al., 2015; Vayron et al., 2016; Dorogoy et al., 2020; Kwak et al., 2020) have been used to understand wave propagation in a dental implant but considered the BII as fully bonded, neglecting the effect of partial contact and of surface roughness. More recently, a finite element model was developed in the time domain to investigate the sensitivity of the

80 ultrasonic response to surface roughness properties of the BII and to osseointegration processes (Hériveaux et al., 2018; Hériveaux et al., 2019a; Hériveaux et al., 2020). The implant roughness was first modeled with an idealized sinusoidal profile (Hériveaux et al., 2018) and actual implant roughness profiles (Hériveaux et al., 2019a), and the 3D case was eventually considered (Hériveaux et al., 2020). However, it still remains difficult to perform the inversion of the 85 ultrasound signal in order to determine the biomechanical properties of the BII. Moreover, the computation cost of the time-domain finite element simulation is still high, thus preventing constructing a sufficiently large database to run data-driven approaches.

Data-driven methods are powerful approaches to solve the inverse problem and have 90 significant advantages because they can be adapted to various conditions by using annotated largescale dataset, which requires detailed understanding of the parametric sensitivity. Artificial neural networks with supervised learning have been used successfully in many engineering domains (Khan et al., 2001; Silver et al., 2016). In particular, the convolutional neural network (CNN) method showed an excellent performance in terms of image recognition and classification 95 (Krizhevsky et al., 2012; Simonyan and Zisserman, 2014). Moreover, various applications of the CNN method have been presented including structural health monitoring and source localization (Kwak, 2018; Toh and Park, 2020). However, collecting large-scale dataset in the implant research field is not straightforward unlike measuring visual data such as images. CNNs with artificial 
imaging processes such as coloring, distorting and noising were presented to augmented training 100 dataset numerically (Antoniou et al., 2017; Perez and Wang, 2017; Salamon and Bello, 2017; Kwak et al., 2021). Inspired by researches for the image recognitions, the spectrogram and spacetime matrices were employed to convert input acoustic data into relevant two dimensional color figures (Costa et al., 2017; Vesperini et al., 2018).

The aim of this numerical study is to assess osseointegration phenomena by inverting ultrasonic signals using a 2-D convolutional neural network (CNN). Osseointegration phenomena are determined based on the estimation of the soft tissue thickness present at the BII that is obtained by analyzing the interaction of the ultrasonic waves with the BII. To do so, the forward model was modified compared to (Hériveaux et al., 2018; Hériveaux et al., 2019a; Hériveaux et al., 2020)

110 since it was derived in the frequency domain and then translated into the time domain, which has the advantage of significantly reducing the computation cost, allowing to construct an annotated large dataset. The $\mathrm{CNN}$ based-assessment method was then applied to the aforementioned dataset to assess the osseointegration at the microscopic and macroscopic roughness scales. Our algorithm was verified by separating the validation dataset from the training dataset.

\section{Material and methods}

\section{A. Description of the problem}

We consider a two-dimensional model which consists of three domains, corresponding to 120 the implant (Titanium alloy, Ti-6Al-4V), cortical bone tissue and soft tissue (see Fig. 1a). The incident wave is assumed to be as a pulsed plane wave propagating from up-to-down vertical direction. Following the in vitro experiment setup described in (Mathieu et al., 2012), the objective is to determine the radiofrequency signal corresponding to the wave reflected by the BII.

After the implant insertion, collagen-rich tissues form around the implant and are then mineralized, which corresponds to a transformation from soft tissue into a solid material during osseointegration phenomena (Moerman et al., 2016). Based on this transformation, we assumed that the soft tissue thickness decreases when osseointegration phenomena progress, which is a similar model than that used in (Raffa et al., 2019; Raffa et al., 2020) to model osseointegration phenomena in the static regime. Moreover, we have shown experimentally that the ultrasound 
130 reflection coefficient of the BII is very sensitive to the properties of bone tissue located around the BII (Mathieu et al., 2012; Hériveaux et al., 2019; Fraulob et al., 2020).

Due to the symmetry of the problem, the streamlined model described in Fig. 1 was introduced similarly as in our previous studies (Hériveaux et al., 2018; Hériveaux et al., 2019a; Hériveaux et al., 2020). Only a single half-sine period of the interface was considered (Figure 1b),

135 which is sufficient to simulate the propagation of the acoustic wave using symmetrical boundary conditions in the interfaces perpendicular to the direction $x$. The implant surface roughness was modeled by a sinusoidal function of amplitude $h$ and half-period $L$.

The interface roughness was analyzed by considering two different scales: denoted 140 microscopic and macroscopic roughness in what follows. The microscopic roughness corresponds to the situation obtained by sandblasting and acid etching (Strnad and Chirila, 2015) of the implant surface, while the implant geometries such as threading of dental implant and pedicle screws correspond to the macroscopic roughness. For the microscopic case, the reference values of the surface roughness amplitude $h$ and half-period $L$ are $5 \mu \mathrm{m}$ and $50 \mu \mathrm{m}$, respectively (Hériveaux et 145 al., 2018). For the macroscopic case, the reference values of the surface roughness amplitude $h$ and half-period $L$ are $360 \mu \mathrm{m}$ and $900 \mu \mathrm{m}$, respectively (Hériveaux et al., 2018).

Soft tissue was considered between the bone and the implant (see Figure 1a) in order to model non-mineralized fibrous tissue that may be present at the BII in the case of non150 osseointegrated implants or just after surgery. The soft tissues thickness $W$ was defined as the distance between the highest point of the implant profile and the bone layer (see Figure 1b). The progression of the osseointegration phenomena leads to a decrease of the soft tissue thickness $W$. 


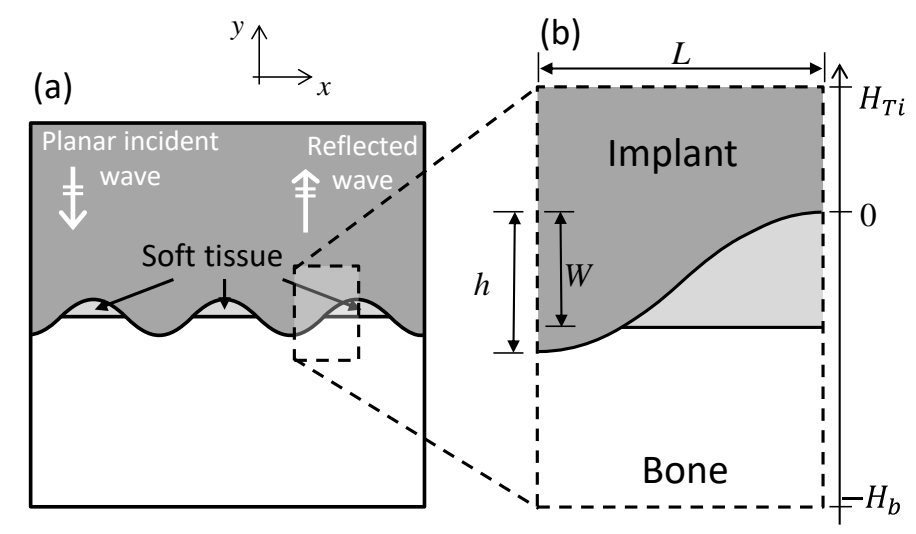

155 FIG 1. (a) Schematic illustration of the geometrical configuration of the BII considered in the numerical simulation. (b) Description of the streamlined model.

The size of the implant and bone domains ( $H_{b}$ and $H_{T i}$, respectively) were much larger than the characteristic lengths of the interface roughness so that they may be considered as semi-infinite domains. Lengths of the implant $H_{T i}$ and cortical bone $H_{b}$ domains in vertical direction are $1 \mathrm{~mm}$ in the macroscopic case and $50 \mu \mathrm{m}$ in the microscopic case, which corresponds to two times the wavelength in the $y$ direction. In this study, we assumed that all materials have linear isotropic elastic behavior. The material properties of all media considered in the numerical simulation were taken from (Njeh et al., 1999; Pattijn et al., 2006; Pattijn et al., 2007; Haïat et al., 2009) (Table I).

Table I. Material properties used in the numerical simulations. Values taken from (Njeh et al., 1999; Pattijn et al., 2006; Pattijn et al., 2007; Haïat et al., 2009).

\begin{tabular}{lccc}
\hline \hline & $C_{p}\left(\mathrm{~m} \cdot \mathrm{s}^{-1}\right)$ & $C_{s}\left(\mathrm{~m} \cdot \mathrm{s}^{-1}\right)$ & $\rho\left(\mathrm{kg} \cdot \mathrm{m}^{-3}\right)$ \\
\hline Soft tissue (liquid) & 1500 & 10 & 1000 \\
Titanium & 5810 & 3115 & 4420 \\
Cortical bone tissue & 4000 & 1800 & 1850 \\
\hline \hline
\end{tabular}

\section{B. Time-domain computation of the reflection coefficient of the bone-implant interface}

In this study, we are interested in the determination of the reflection coefficient of the BII that is evaluated based on the amplitudes of the incident and reflected waves in the time-domain. 
As it has been done experimentally, the average amplitude of the reflected waves was evaluated 175 over a horizontal surface located at a given distance from the BII.

To solve the aforementioned problem, the approach developed in our previous studies (Hériveaux et al., 2018; Hériveaux et al., 2019a; Hériveaux et al., 2020) consisted of i) considering a finite element discretization used to derive the system of dynamic equations and ii) using direct time integration schemes. The finite element simulation was performed by using the Comsol Multiphysics software (COMSOL AB, Stockholm, Sweden) using the $\alpha$-generalized scheme. However, such an approach involving a direct time-integration is computationally expensive, hampering their implementation into deep learning-based analyses because of the significant number of realizations required. To reduce computation time, another computational procedure is proposed in what follows. The dynamic problem is firstly solved in the frequency domain, and the solution of reflected wave is then reconstructed in the time domain using the inverse Fourier transform.

\section{Resolution in the frequency domain}

For each subdomain $\# i$ ( $i=1,2,3$ correspond to titanium implant, soft tissues and cortical bone respectively) defined in the previous subsection, the dynamic equations in the frequency domain read:

$$
-\omega^{2} \rho^{(\mathrm{i})} \boldsymbol{u}^{(i)}-\operatorname{div} \boldsymbol{\sigma}^{(\mathrm{i})}=\mathbf{0}
$$

where $\rho$ is the mass density, $\boldsymbol{u}$ is the displacement vector and $\boldsymbol{\sigma}$ is the stress tensor in the 195 subdomain \#i. The constitutive relation using Hooke's laws is given by:

$$
\boldsymbol{\sigma}^{(\mathrm{i})}=\lambda^{(\mathrm{i})} \operatorname{tr}\left(\boldsymbol{\varepsilon}^{(\mathrm{i})}\right) \boldsymbol{I}+2 \mu^{(\mathrm{i})} \boldsymbol{\varepsilon}^{(\mathrm{i})},
$$

where $\varepsilon=\frac{1}{2}\left(\operatorname{grad} \boldsymbol{u}+\operatorname{grad} \boldsymbol{u}^{T}\right)$ is the strain tensor in the subdomain \#i.

The continuity of displacement and stress fields lead to the following boundary conditions at 200 the interface between subdomain $\# i$ and $\# k$ :

$$
\left\{\begin{aligned}
\boldsymbol{u}^{(j)} & =\boldsymbol{u}^{(k)} \\
\boldsymbol{\sigma}^{(i)} \boldsymbol{n} & =\boldsymbol{\sigma}^{(k)} \boldsymbol{n}
\end{aligned} \quad \text { at interface }(i-k)\right.
$$

where $\{i, k\}=\{1,2\},\{1,3\}$ or $\{2,3\}$ and $\boldsymbol{n}$ is the unitary vector normal to the interface. 
Here, we considered an incident wave propagating in the $y$-direction, perpendicularly to 205 the BII (see Figure 1). The displacement field $\boldsymbol{u}^{(1)}$ in the subdomain \#1 can be decomposed into two parts representing the incident and reflected wave fields as follows:

$$
\boldsymbol{u}^{(1)}=\boldsymbol{u}^{I}+\boldsymbol{u}^{R}
$$

where $\boldsymbol{u}^{I}=\left\{0, U^{I} e^{j k_{p}^{(1)} y}\right\}$ in which $k_{p}^{(1)}$ and $U^{I}$ are the wavenumber and the amplitude of the incident P-wave, respectively; $k_{p}^{(1)}=\omega / c_{p}^{(1)}$. As $\boldsymbol{u}^{I}$ is supposed to be known, we substituted Eq.

210 (4) into Eqs. (1-2) to formulate a system of equations with respect to $\boldsymbol{u}^{R}$ as:

$$
\begin{gathered}
-\omega^{2} \rho^{(1)} \boldsymbol{u}^{R}-\operatorname{div} \boldsymbol{\sigma}^{R}=\mathbf{0}, \\
\boldsymbol{\sigma}^{R}=\lambda^{(1)} \operatorname{tr}\left(\boldsymbol{\varepsilon}^{R}\right) \boldsymbol{I}+2 \boldsymbol{\mu}^{(1)} \boldsymbol{\varepsilon}^{R}
\end{gathered}
$$

In Eqs. (5-6), all the terms related to $\boldsymbol{u}^{I}$ did not appear because $\boldsymbol{u}^{I}$ is also a solution of the 215 dynamic equation (1). However, $\boldsymbol{u}^{I}$ cannot be neglected in the continuity conditions which are now expressed by:

$$
\begin{gathered}
\boldsymbol{u}^{R}+\boldsymbol{u}^{I}=\boldsymbol{u}^{(k)} \\
\left(\boldsymbol{\sigma}^{R}+\boldsymbol{\sigma}^{I}\right) \boldsymbol{n}=\boldsymbol{\sigma}^{(k)} \boldsymbol{n}
\end{gathered} \quad \text { at intefaces }(1-k) \text { with } k=\{2,3\}
$$

where:

$$
\boldsymbol{\sigma}^{I}=\left[\begin{array}{cc}
2 j k_{p}^{(1)} \mu^{(1)} U^{I} e^{j k_{p}^{(1)} y} & 0 \\
0 & j k_{p}^{(1)}\left(\lambda^{(1)}+\mu^{(1)}\right) U^{I} e^{j k_{p}^{(1)} y}
\end{array}\right]
$$

220

The radiation conditions at the infinity read

$$
\begin{array}{r}
\boldsymbol{u}^{R} \rightarrow \mathbf{0} \text { when } y \rightarrow+\infty, \\
\boldsymbol{u}^{(3)} \rightarrow \mathbf{0} \text { when } y \rightarrow-\infty,
\end{array}
$$

225

The system of equations ((Eqs. (5-9)) was implemented using the finite element software COMSOL Multiphysics (Stockholm, Sweden). As the domain's size should be finite, to prevent the non-physical reflected waves from the upper and lower boundaries (Eqs. 8\&9), two absorbing layers are introduced at $y=H_{T i}$ and at $y=-H_{b}$. By introducing absorbing layers, the radiation conditions can be represented artificially, resulting in smoothly attenuated radiative waves. With 
the presence of absorbing layers, at the upper boundary of the implant domain $\left(\Omega^{(1)}\right)$ and at the lower boundary of bone domain $\left(\Omega^{(3)}\right)$, fixed boundaries are imposed:

$$
\begin{gathered}
\boldsymbol{u}^{R}=\mathbf{0} \text { at } y=H_{T i}, \\
\boldsymbol{u}^{(3)}=\mathbf{0} \text { at } y=-H_{b},
\end{gathered}
$$

235 In addition, the symmetry conditions also impose that $u_{x}=0$ at the lateral boundaries $x=0$ and $x=L$.

In each subdomain, the element size was chosen to be equal to $\lambda_{\min } / 20$, where $\lambda_{\min }$ denotes the shortest wavelength in the considered domain (i.e. the wavelength of shear wave). The implant and bone subdomains were meshed by structured quadrangular quadratic elements, and 240 the soft tissue subdomain was meshed with triangular quadratic elements. Solving the system (Eqs. 5-9) leads to the solutions of $\boldsymbol{u}^{\mathrm{R}}, \boldsymbol{u}^{(2)}, \boldsymbol{u}^{(3)}$ as a function of $\boldsymbol{u}^{\mathrm{I}}$ and $\omega$. The evaluation of the reflection coefficient requires calculating the average displacement over a horizontal section of the implant domain which is located at an ordinate $y_{m}$ from the bone-implant interface. In the frequency domain, this average displacement is given by:

$$
\Phi^{R}(\omega)=\frac{1}{L} \int_{0}^{L} U_{2}^{R}\left(x, y_{m}, \omega\right) d x=H(\omega) U^{I}(\omega)
$$

where $H(\omega)$ denotes the transfer function and $U^{I}$ is the amplitude of the incident wave.

\section{Determination of the solution in the time-domain}

The incident plane wave was given by a uniform vertical stress $\sigma_{22}^{I}$ which has a broadband 250 ultrasonic pulse form defined by:

$$
\sigma_{22}^{I}(t)=A e^{-4\left(f_{c} t-1\right)^{2}} \sin \left(2 \pi f_{c} t\right)
$$

where $A$ indicates an arbitrary constant and $f_{c}=10 \mathrm{MHz}$ indicates its central frequency chosen based on the QUS device developed by our group to assess dental implant stability (Mathieu et al., 2011; Vayron et al., 2013; Vayron et al., 2014a; Vayron et al., 2014c; Vayron et al., 2018a; Vayron et al., 2018b). The frequency response corresponding to the average displacement $\Phi^{R}(\omega)$ of the reflected wave at the surface located at $y_{m}$ can be expressed as:

$$
\Phi^{R}(\omega)=H(\omega) S(\omega) /\left(j \omega \rho^{(1)} C_{p}^{(1)}\right)
$$

where $C_{p}^{(1)}$ denotes the compressional velocity in the subdomain \#1 and $S(\omega)$ denotes the Fourier transform of $\sigma_{22}^{I}(t)$ given by $S(\omega)=\int \sigma_{22}^{I}(t) \cdot e^{-j \omega t} d t$. 
Zero-padding was performed in the frequency domain to obtain a high-resolution time response as follow:

$$
\Phi^{*}(n \Delta \omega)=\left\{\begin{array}{cc}
\Phi^{\mathrm{R}}(n \Delta \omega) & 0<n<N-1 \\
0 & N<n<N^{*}-N+1 \\
\Phi^{R}\left(\left(N^{*}-n\right) \Delta \omega\right) & N^{*}-N+2<n<N^{*}-1
\end{array},\right.
$$

where $\Delta \omega$ is the frequency step of the simulation, $N$ is the number of discrete data obtained by the

265 simulation, $N^{*}$ is the extended number of data by the zero-padding. The time-domain response with higher resolution $s(t)$ can be calculated by the inverse Fourier transform of $\Phi^{*}$. Notably, the frequency bandwidth analyzed herein was limited to $30 \mathrm{MHz}$ and the frequency step of the numerical model was set to $0.5 \mathrm{MHz}(N=60)$, resulting in $2 \mu$ s for the time duration and $33.3 \mathrm{~ns}$ for the time step. Considering that a tight time step is necessary for a satisfactory numerical 270 analysis for the BII (Hériveaux et al., 2018), the extended number $N^{*}$ was set to 840 to increase time resolution of the response by 14 times, resulting in $28 \mu \mathrm{s}$ for the time duration and $2.4 \mathrm{~ns}$ of the adjusted time step. Accordingly, we obtained the high-resolution time response efficiently from only 60 simulated data in the frequency domain, while the simulation based on the direct timeintegration would be computationally more expensive.

275

The reflection coefficient $R$ was determined based on the ratio of the amplitudes of the reflected and incident waves in the time domain. The first signal corresponds to the uniform incident vertical displacement, noted $s_{i}(t)$. The second signal corresponds to $s_{r}(t)$. The maximum amplitude of the modulus of the Hilbert's transform (corresponding to the envelope) of $s_{i}(t)$ and $s_{r}(t)$ were noted $A_{i}$ and $A_{r}$, respectively. The reflection coefficient $R$ in amplitude then writes:

$$
R=\frac{A_{r}}{A_{i}}
$$

\section{E. Composition of the training dataset}

To synthesize the training dataset, the ultrasound simulations were performed with the 285 range of variation of the BII roughness parameters, considering the results obtained from our previous studies (Hériveaux et al., 2018; Hériveaux et al., 2019a; Hériveaux et al., 2020). In the case of the microscopic roughness, the training data set was constructed with various values of $h$ comprised between 0 and $10 \mu \mathrm{m}$ with a step of $1 \mu \mathrm{m}$ and of $W$ comprised between 0 and $100 \mu \mathrm{m}$ 
with a step of $0.5 \mu \mathrm{m}$. In the case of the macroscopic roughness, the training data set was 290 constructed with various values of $h$ comprised between 310 and $410 \mu \mathrm{m}$ with a step of $10 \mu \mathrm{m}$ and of $W$ comprised between 0 and $1.5 h$ with a step of $0.01 h$. To employ separate training and validation dataset, all reference cases for both roughness scales $(h=5 \mu \mathrm{m}$ for the microscopic scale and $h=360 \mu \mathrm{m}$ for the macroscopic scale) were excluded from the training dataset. The excluded dataset was then employed only to validate our method, resulting in a ratio of the validation data to the training data equal to 0.1 .

The ultrasound radiofrequency signals obtained using the numerical model for each set of parameters $(h, W)$ corresponding to a given roughness and state of osseointegration were transformed into time-frequency spectrograms using short-time Fourier transform (STFT). In this study, the spectrograms were used as the input data of the 2-D CNN. We employed the rectangular window function for calculation of the spectrograms, which are not post-processed to reflect the magnitude of the radio frequency signals as they are. Considering the resolution of the spectrogram in time and frequency domains, we chose the window size and overlap ratio to be $2^{7}$ and $7 / 8$, respectively, resulting in $0.04 \mu \mathrm{s}$ time step and $3.125 \mathrm{MHz}$ frequency step. Moreover, since the emitted signal is a pulse wave with a center frequency of $10 \mathrm{MHz}$ (see Figure 3), the spectrogram above $1 \mu \mathrm{s}$ and $37.5 \mathrm{MHz}$ in time and frequency domain respectively were excluded from the analysis.

\section{F. Layer configuration of the 2-D convolution neural network}

The layer structure of the 2-D CNN employed herein is illustrated in Figure 2. The structure consists of two convolution layers. We used the Adam optimization algorithm for training our network because it requires little hyperparameter tuning effort for achieving adequate results (Kingma and $\mathrm{Ba}, 2014$ ). A mini-batch size was 120, and 100 epochs were conducted. 32 convolution filters were used in the first and the second convolution layer. The size of the

315 convolution kernel was 4 by 4 with strides of two. To prevent change of the size of images, zeros were added at edges(Chetlur et al., 2014). The batch normalization was performed with each convolution layer(Ioffe and Szegedy, 2015). The rectified linear unit (ReLU) was used as the activation function(Nair and Hinton, 2010). The max pooling layer was performed with a 4 by 4 matrix and strides of two. After the second max pooling layer, $10 \%$ of neurons were dropped out 
320 to prevent over-fitting(Hinton et al., 2012). The flatten-layer was used before the first fully connected layer. The layer structure ends with two fully connected layers, which have 32 neurons and 1 neuron, to obtain the estimated soft tissue thickness $W^{e}$.

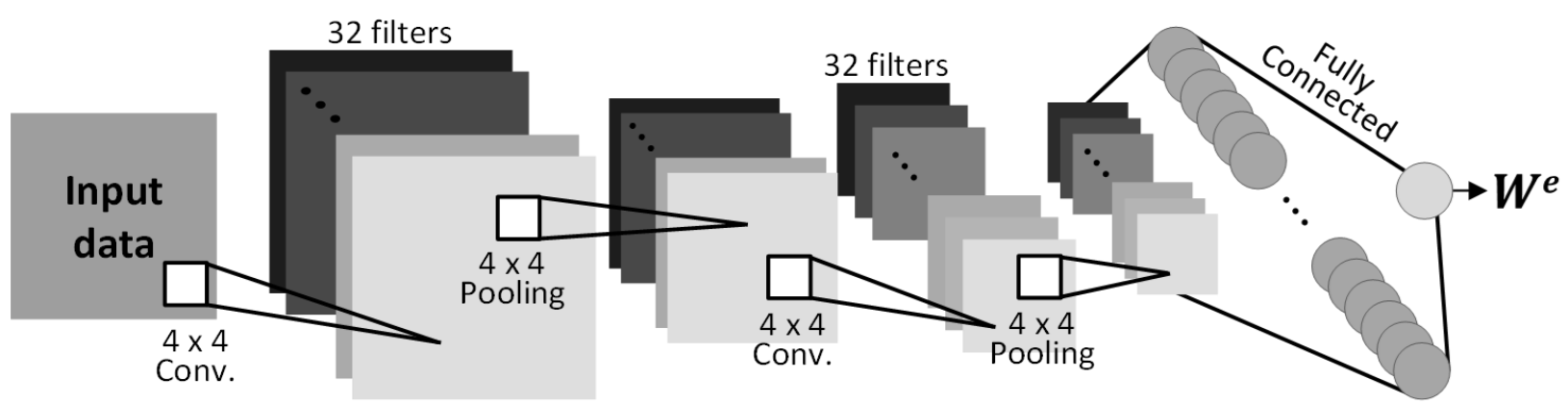

FIG 2. Layer structure of the proposed CNN to assess the soft tissue thickness $W$. $W^{e}$ corresponds to the estimated soft tissue thickness.

\section{G. Examination of the proposed assessment network}

The convolution network learns salient features to classify the input data during the training 330 process. Each pixel of input images into the network is transformed based on the weights of neurons in the network layers determined by training of the large-annotated dataset. As implied by the "hidden" layer on neural networks, it may be difficult to know what features are trained exactly or how well the network will work. The feature map for a specific input can provide visualized information to understand what features in the input play a key role through the network (Yosinski et al., 2015). In other words, we can examine the reliability of networks from the visualized information. To examine the training process with the dataset built by the BII forward model, the feature map of each convolution layer was analyzed for the reference roughness cases of both scales and compared between different soft tissue thickness cases.

The bias of the estimation and the $95 \%$ limits of agreement (LOA), which has been widely used for evaluating regression results (Park et al., 2020), were computed to examine the estimated soft tissue thickness by the proposed assessment network. The bias $\bar{m}$ corresponds to the averaged value of the differences between the estimated and actual soft tissue thicknesses. The 95\% LOA is defined as: 


$$
\mathrm{LOA}=\bar{m} \pm 1.96 \sqrt{\sum_{i=1}^{n}\left(d_{i}-\bar{m}\right)^{2} /(n-1)}
$$

where $d$ is the difference between the estimated and actual soft tissue thicknesses, and $n$ is the total number of validation dataset. The linear correlation $R$ between two thicknesses are evaluated such that:

$$
R=E\left[\left(W_{a}-E\left(W_{a}\right)\right)\left(W_{e}-E\left(W_{e}\right)\right)\right] / \sqrt{E\left[\left(W_{a}-E\left(W_{a}\right)\right)^{2}\right] \cdot E\left[\left(W_{e}-E\left(W_{e}\right)\right)^{2}\right]}
$$

350 where $\mathrm{E}(\ldots)$ indicates an averaged value, $W_{a}$ and $W_{e}$ are the actual and estimated values of the soft tissue thickness, respectively.

\section{Results}

\section{A. Validation of the forward model}

Figure 3 shows the comparison between the radiofrequency signals corresponding to the ultrasound responses of the bone-implant interface for $h=360 \mu \mathrm{m}$ and $L=900 \mu \mathrm{m}$ (macroscopic case) and obtained using the proposed model (in the frequency domain) and using the method described in (Hériveaux et al., 2018) (in the time domain). A good agreement is obtained between the two models, which constitutes a validation of the forward model.
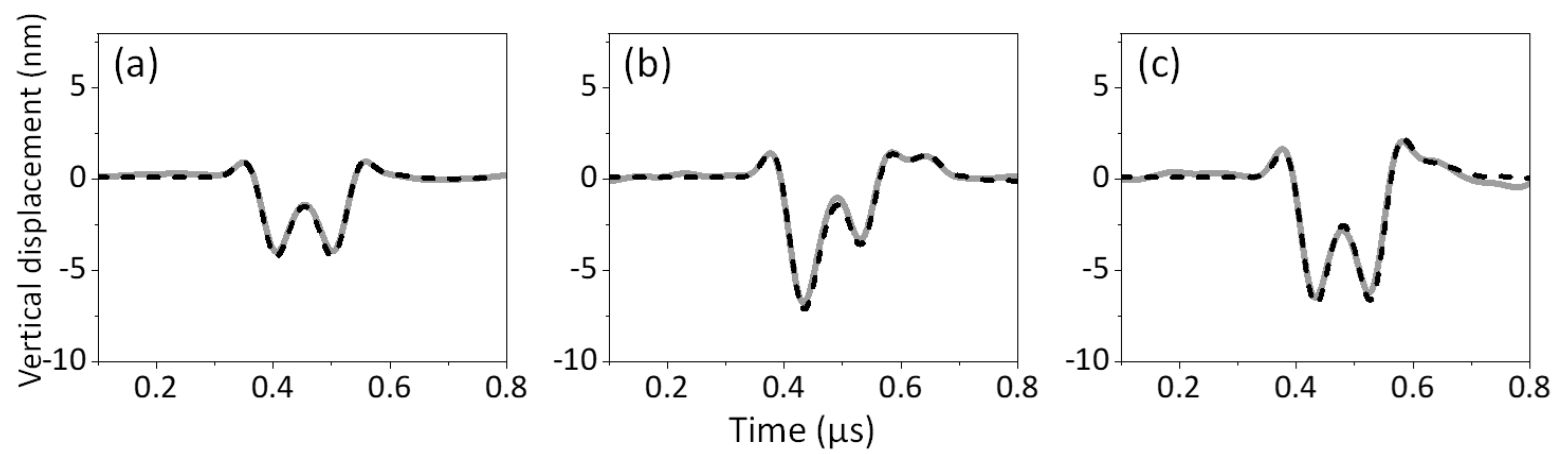

365 FIG. 3. Radiofrequency signals corresponding to the ultrasound responses of the bone-implant interface for $h=360 \mu \mathrm{m}, L=900 \mu \mathrm{m}$ (macroscopic case) and $W / h=$ (a) 0 , (b) 0.5 , (c) 1 . The solid 
grey lines indicate the results obtained using the proposed model (in the frequency domain) and the dashed black lines indicate the results obtained in (Hériveaux et al., 2018) (in the time domain).

Figure 4 shows the variation of the reflection coefficient $R$ for different values of the roughness amplitude $h$ as function of the soft tissue thickness $W$ in the microscopic case ( $L=50$ $\mu \mathrm{m})$ and as a function of $W / h$ in the macroscopic case $(L=900 \mu \mathrm{m})$. The dashed lines correspond to the results obtained in (Hériveaux et al., 2018) (in the time domain) and the symbols indicate the results obtained using the proposed model in the frequency domain. As shown in Figure 4, a good agreement is obtained between the two models. The average relative error is equal to $1.8 \%$ in the microscopic case and to $1.4 \%$ in the macroscopic case, which may be explained by numerical errors. Again, Figures $3 \& \mathbf{4}$ clearly support the validity of the numerical model developed herein.
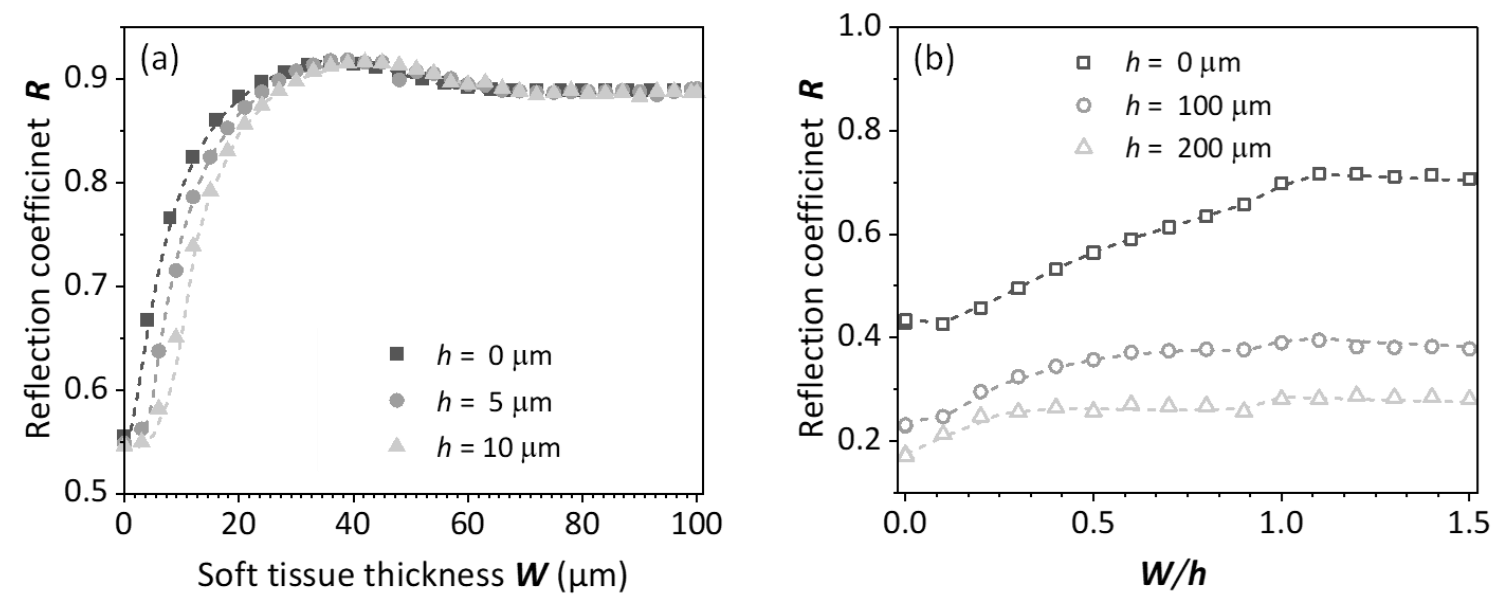

FIG. 4. Variation of the reflection coefficient $R$ for different values of the roughness amplitude $h$ as function of (a) the soft tissue thickness $W$ in the microscopic case $(L=50 \mu \mathrm{m})$ and (b) the ratio of the soft tissue thickness $W$ and of the roughness amplitude $h$ in the macroscopic case $(L=900$ $\mu \mathrm{m})$. The symbols indicate the result obtained by the proposed model in the frequency domain for 385 the different values of $h$, and the dashed lines indicate the corresponding result obtained using the time-domain model in (Hériveaux et al., 2018). 


\section{B. Spectrogram of the rf signals}

Figure 5 shows the spectrograms of the signal corresponding to the reflection of the 390 ultrasonic wave on the BII when the soft tissue thickness varies in the microscopic case (Figures 5a-d) and in the macroscopic case (Figures 5e-h). Each spectrogram exhibits a different pattern according to the value of the soft tissue thickness, which demonstrates that the spectrograms are sensitive to $W$. The sensitivity of the spectrogram pattern indicates that CNN is likely to learn features in the time and frequency domains simultaneously from the ultrasound reflections.

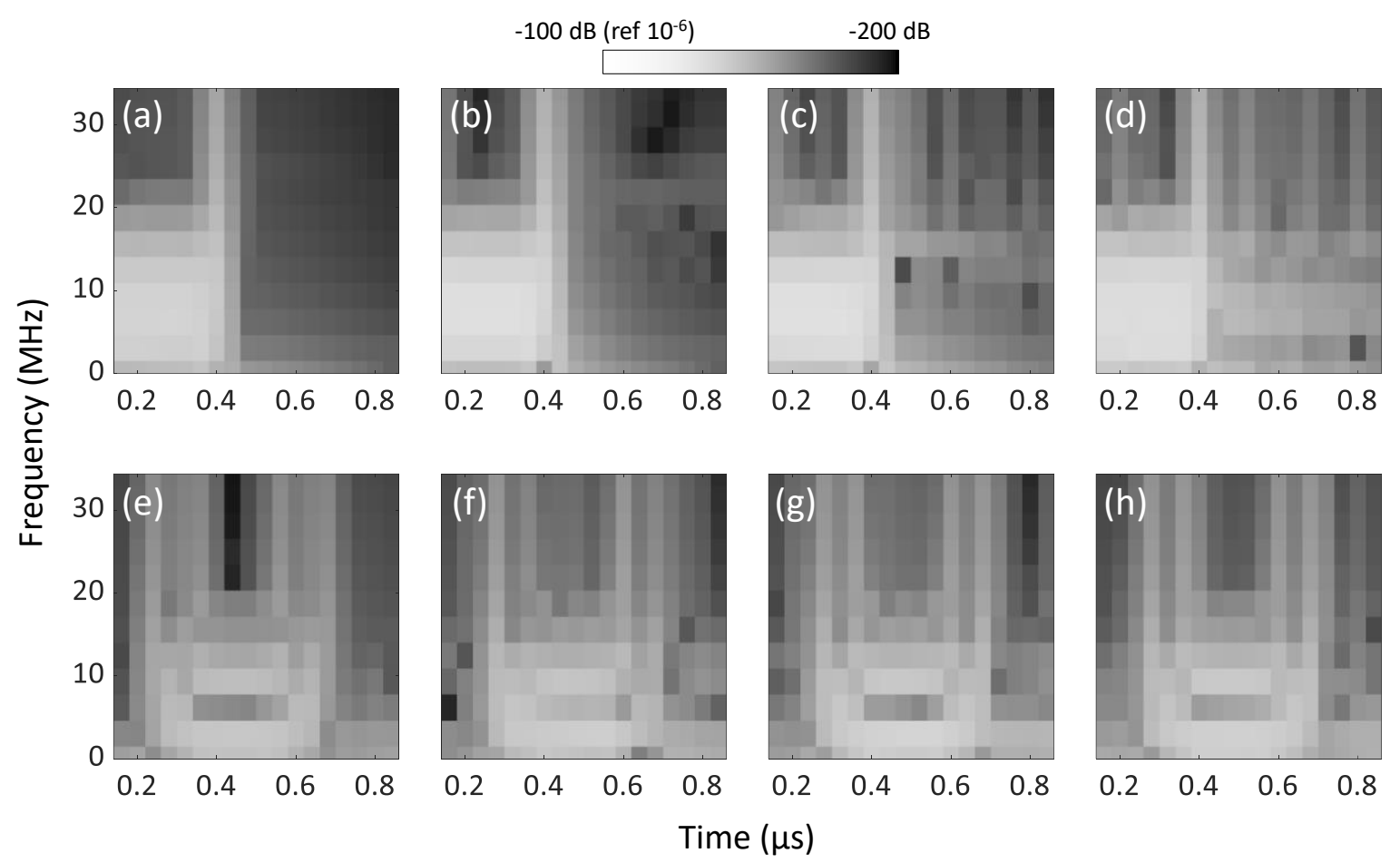

FIG. 5. Spectrograms of the ultrasonic signals as a function of the soft tissue thickness. (a)-(d) For the microscopic case, $h=5 \mu \mathrm{m}, L=50 \mu \mathrm{m}$ and (a): $W=0 \mu \mathrm{m}$, (b): $25 \mu \mathrm{m}$, (c): $50 \mu \mathrm{m}$, (d): 100 $\mu \mathrm{m}$. (e)-(h) For the macroscopic case, $h=360 \mu \mathrm{m}, L=900 \mu \mathrm{m}$ and (e): $W / h=0$, (f): 0.5, (g): 1.0, $400 \quad$ (h): 1.5.

\section{Feature map of the proposed network}

We looked over the feature map of the convolutional layer for the reference cases of the microscopic and macroscopic roughness cases, which was excluded from the training dataset. For 405 the microscopic roughness, the two representative cases corresponding to $W=50,100 \mu \mathrm{m}$ were 
employed as the input data to derive the corresponding feature maps. Since the two convolutional layers have 32 filters, Figures 6a-h show the 32 output channels for each convolutional layer and each configuration arranged sequentially from left to right. The first convolutional layers corresponding to the microscopic case and to a change of the osseointegration phenomena ( $W=50$

410 and $100 \mu \mathrm{m}$, Figures $\mathbf{6 a} \& \mathbf{b})$ look similar, suggesting that the first layer is insufficient to classify the target in a satisfactory manner. However, based on the analysis of the second convolutional layers corresponding to the microscopic case (Figures $6 \mathbf{c} \& \mathbf{d}$ ), although the outputs became more abstract, the feature maps between the two representative cases can be clearly distinguished, indicating that the proposed network successfully capture the inherent features from the BII 415 reflections to classify osseointegration phenomena.

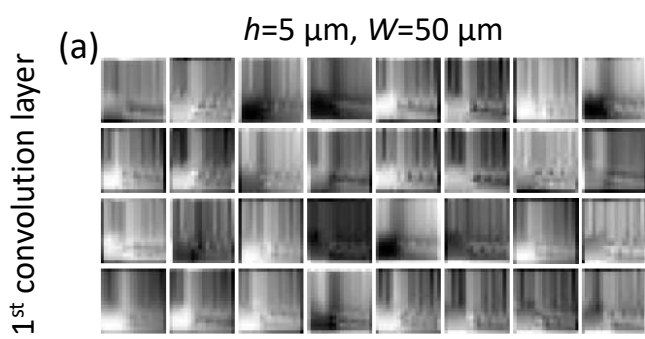

(b)
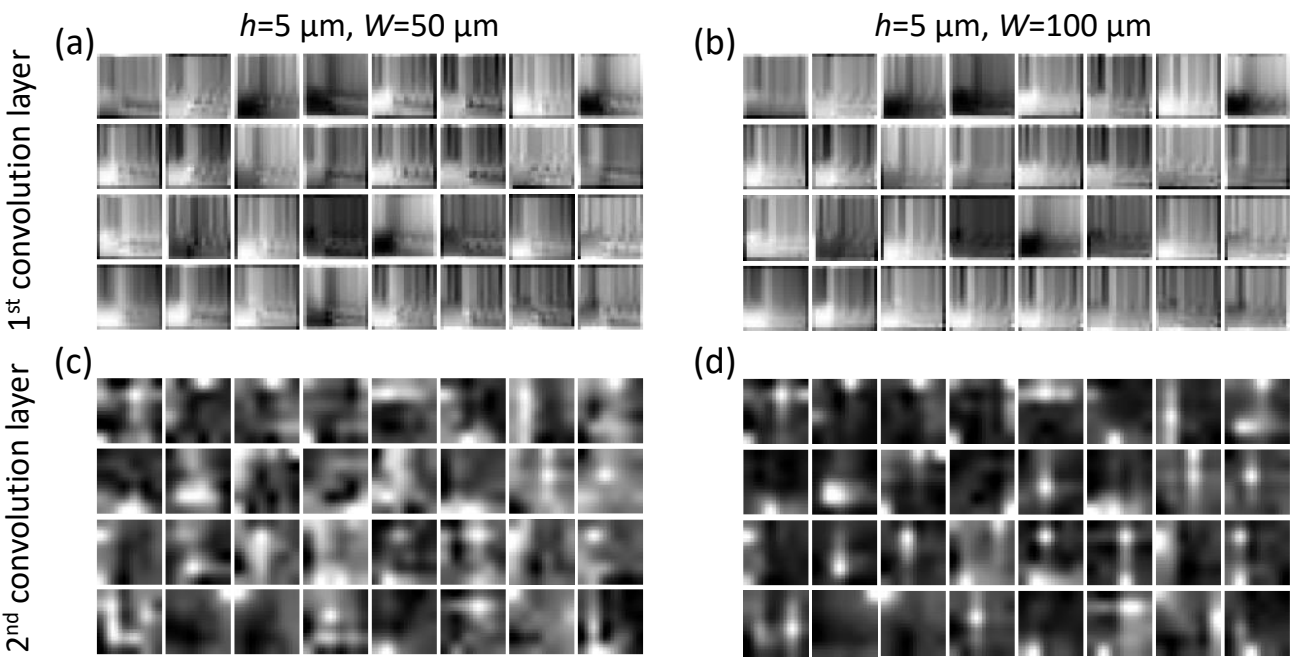

(d)
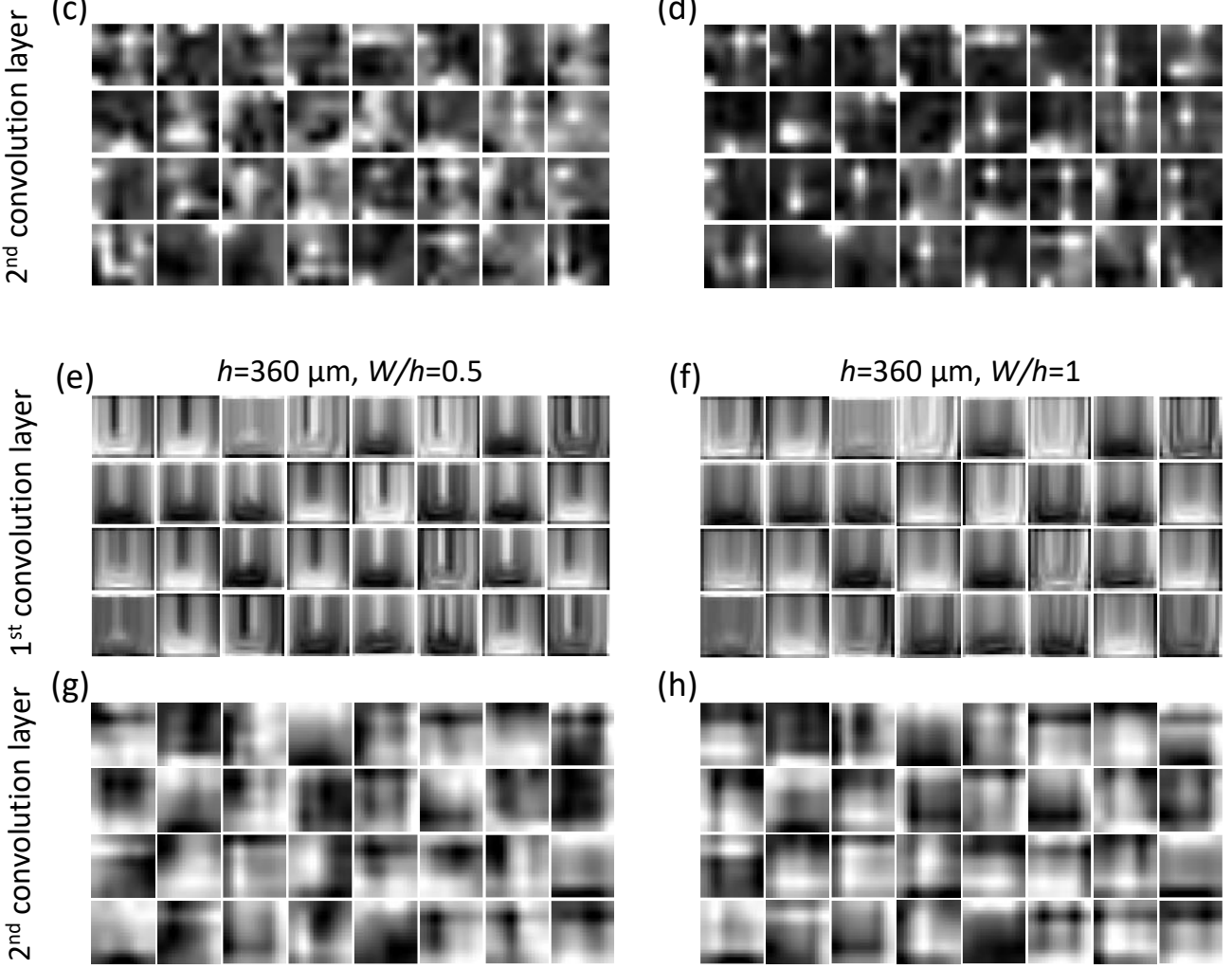

(f)



(h)

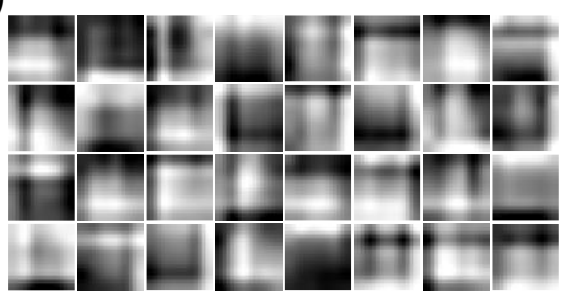


FIG. 6. Representative feature maps of the convolution layers for the reference BII roughness cases, separated from the training dataset. (a)-(d) For $h=5 \mu \mathrm{m}, L=50 \mu \mathrm{m}$ for the microscopic roughness, the results from the first and the second convolution layers for the case of $W=50 \mu \mathrm{m}$

420 ((a)\&(c), respectively) and $100 \mu \mathrm{m}$ ((b)\&(d), respectively). (e)-(h) For $h=360 \mu \mathrm{m}, L=900 \mu \mathrm{m}$ for the macroscopic roughness, the results from the first and the second convolution layers for the case of $W / h=0.5((\mathrm{e}) \&(\mathrm{~g})$, respectively) and $1.0((\mathrm{f}) \&(\mathrm{~h})$, respectively).

Likewise, two representative cases of the macroscopic roughness $(W / h=0.5$ and 1.0$)$ were considered. As expected, the feature maps from the first convolutional layer provide relatively similar results to the inputs (Figures $6 \mathbf{6} \& \mathbf{f}$ ), whereas clear differences are found between the two maps from the second convolutional layer (Figures $6 \mathbf{g} \& \mathbf{h}$ ). The representative feature maps clearly demonstrate the reliability of our network for the microscopic and macroscopic roughness, trained with the signals from the proposed forward model.

\section{Assessment of osseointegration phenomena status}

Figure 7a (respectively 7b) shows the comparison between the actual value and the estimation of the soft tissue thickness for the microscopic (respectively macroscopic) roughness case. The linear correlation between actual and estimated values of the soft tissue thickness shows excellent $R^{2}$ values equal to $99.53 \%$ for the microscopic roughness and $99.65 \%$ for the macroscopic roughness. For the case of the microscopic roughness, the bias computed with the estimated values was equal to $0.88 \mu \mathrm{m}$, and the LOA corresponded to [-2.56, $4.32 \mu \mathrm{m}]$. For the case of the macroscopic roughness, the bias computed with the estimated values was equal to 7.30 $\mu \mathrm{m}$, and the LOA corresponded to $[-15.75,30.35 \mu \mathrm{m}]$. These results show the reliability of the proposed assessment of osseointegration phenomena. 

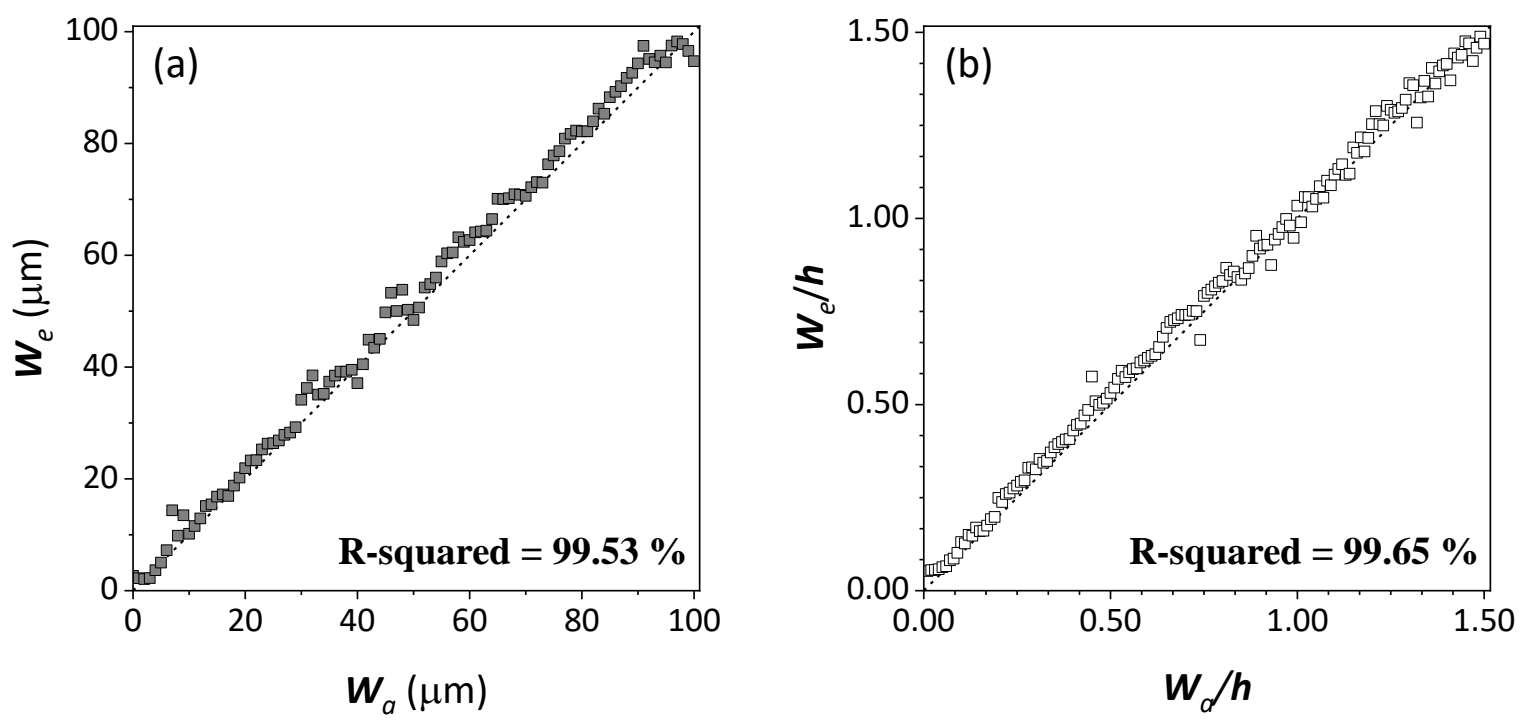

FIG. 7. Comparison between the actual and estimated values of the soft tissue thickness using the proposed convolutional neural network applied to the ultrasound reflections on the BII for (a) $h=$ $5 \mu \mathrm{m}, L=50 \mu \mathrm{m}$ (the microscopic roughness), (b) and $h=360 \mu \mathrm{m}, L=900 \mu \mathrm{m}$ (the macroscopic 445 roughness).

\section{Discussion}

A large-scale annotated dataset with diverse conditions should be considered to employ deep learning methods. However, accumulating a large-scale dataset in biomechanical engineering may be difficult because i) of the difficulty to obtain an important number of samples due to ethical

450 requirements and ii) it is impossible to control all parameters affecting the ultrasound response of the BII since they vary simultaneously (Fraulob et al., 2020) as a function of healing time. Here, we present a method based on the deep convolutional neural network to assess osseointegration phenomena. The method is trained with a synthesized dataset corresponding to numerical results. The originality of the proposed approach lies on hybridizing CNN and numerical simulation in

455 order to assess osseointegration phenomena. Our method relies on the estimation of soft tissue thickness in the microscopic and macroscopic roughness cases based on the analysis of the signal corresponding to the reflection of an ultrasonic wave on the BII. To do so, a numerical model of the interaction between an ultrasonic wave and the BII has been validated by comparison with a model developed previously in the time domain (Hériveaux et al., 2018; Hériveaux et al., 2019a; 460 Hériveaux et al., 2020). The advantage of the present numerical model is that i) only 60 simulated 
data in the frequency domain are needed to obtain the signal in the time domain and ii) a single transfer function of the BII can be used to obtain simulated reflections for different acoustical sources by a simple convolution calculation instead of simulations for all cases. The computation time necessary to simulate the ultrasonic response of the BII in the microscopic (respectively 465 macroscopic) case using the model developed herein is equal to $40 \mathrm{sec}$ (respectively 3 min), which allows us to form the large-annotated training dataset with affordable time cost, whereas $12 \mathrm{~min}$ (respectively 52 min) were needed with our previous model (Hériveaux et al., 2018) in the time domain.

Although the training dataset employed for the proposed data-driven approach is relatively small (3600 computations), the reliability of the method was examined by employing an independent validation dataset. The estimations (Figure 7) were implemented with the dataset of the reference surface roughness cases, which was excluded from the training process. Moreover, the results obtained for both scales exhibit excellent $R^{2}$ values (99.53 and 99.65\%) and narrow

475 LOAs $([-2.56,4.32 \mu \mathrm{m}]$ and $[-15.75,30.35 \mu \mathrm{m}])$ supports the reliability of the proposed CNN method for assessing the effects of osseointegration phenomena. More importantly, the numerical approach under consideration can be used as a complementary tool to compose the data-driven approach for implant clinical studies, which remains difficult to access using experimental approaches only.

Experimental approaches have already shown that the reflection coefficient of the BII is sensitive to healing time (Mathieu et al., 2012), to the implant surface roughness (Fraulob et al., 2020) as well as to the mechanical loading applied to the BII (Hériveaux et al., 2019b). Based on the aforementioned experimental results, an experimental set-up was developed to assess dental 485 implant primary and secondary stability (Vayron et al., 2014a; Vayron et al., 2014c; Vayron et al., 2018a; Vayron et al., 2018b). However, although ultrasound techniques show great potentiality to evaluate dental implant stability, it remains difficult to control the parameters varying simultaneously during osseointegration phenomena, hampering its practical applications. Furthermore, no inversion approach had been carried out and the present study shows the 490 feasibility of applying a model-based inversion technique by using CNN to retrieve quantitative information on the BII. Such an approach should be applied to experimental data in the future. 
Bone (and newly formed bone) tissues are complex materials which may be modeled as heterogeneous, anisotropic viscoelastic and poroelastic media. In the present work, which is the 495 first tentative use of CNNs method in the context of BII characterization using ultrasound, we chose to use the simplest model to describe the bone mechanical behavior because we aim at focusing on the BIC ratio, which is the main parameter of interest. A more detailed description of bone behavior (and in particular taking into account bone anisotropy) would require a much larger set of parameters in the model, and consequently, will increase significantly computational cost.

500 Moreover, it is expected that bone anisotropy has a relatively weak effect of the ultrasound response of the BII because ultrasound at $10 \mathrm{MHz}$ is highly attenuated in bone (Sasso et al., 2007; 2008) and should not propagate over a large distance.

This study has several limitations. First, a 2-D sinusoidal function is used to describe the 505 implant surface roughness, similarly to what was done in (Hériveaux et al., 2018), which constitutes an important approximation allowing to reduce the number of parameters affecting the ultrasonic response of the BII. However, this sinusoidal description may be considered as realistic in the macroscopic case because it models implant threading present in dental implants specifically. For the microscopic case, it has been shown that an actual implant surface profile may be replaced

510 by a sinusoidal profile without modifying significantly the dependence of its ultrasonic response as a function of the soft tissue thickness (Hériveaux et al., 2019a). Although considering 3-D surface function would be more relevant to describe the ultrasound response of the BII, the computational cost required to perform the direct simulations using the 3-D model is significantly higher than for 2-D simulation. Moreover, we have shown that the 2-D approximation may also

515 be considered as realistic compared to the 3-D case(Hériveaux et al., 2020). The two aforementioned studies justify the use of a 2-D sinusoidal description of the implant surface roughness. Second, only normal incidence of the ultrasonic wave is reported, because it corresponds to the experimental situation of interest (Mathieu et al., 2012; Fraulob et al., 2020) and future studies should also consider the normal incidence. Third, this study only considered a 520 center frequency of $10 \mathrm{MHz}$ for the incident wave because it corresponds to the frequency used in the QUS device developed by our group (Vayron et al., 2018a). Fourth, we only considered the 
first reflection of the ultrasound waves on the BII. Analyzing the entire reflection signals (i.e. the coda) would require heavy computation cost and this aspect is left to future studies.

\section{V. Conclusion}

A simulation-based convolutional neural network method was derived in order to assess osseointegration phenomena at the BII. The large-annotated dataset was formed by taking advantage of an efficient ultrasound simulation of the BII. The proposed numerical model in the frequency domain allowed employing numerous simulations efficiently and was validated by a

530 comparison with another model developed in the time domain. The synthesized ultrasound responses were converted into spectrograms and trained to the proposed network structure. The feature maps clearly demonstrated evident differences according to the soft tissue thickness, supporting the reliability of the training process on the proposed network. The reported assessment network for the osseointegration phenomena supports the feasibility of ultrasound analysis to be 535 transformed to noninvasive applications for the bone-implant domain. Moreover, the present approach opens new paths in the development of model-based inversion methods to retrieve the properties of the BII.

\section{Acknowledgements}

540 This project has received funding from the European Research Council (ERC) under the European Union's Horizon 2020 research and innovation program (grant agreement No 682001, project ERC Consolidator Grant 2015 BoneImplant), the National Research Foundation of Korea (NRF) (No. 2019K1A3A1A21031280) and the Partenariat Hubert Curien (PHC) STAR 2019 program (No 43572QM).

\section{Competing interests}

All of the authors declare no competing interests.

\section{Author contributions}

Y.K. developed the analytical model and conducted the analyses. Y.H. and P.B. worked on the finite element analysis. J.P. helped to analyze the neural network. V.H.N. and G.H. supervised 
the overall research. Y.K., V.H.N and G.H. mainly wrote the manuscript. All of the authors discussed the results and the manuscript.

\section{References}

Antoniou, A., Storkey, A., and Edwards, H. (2017). "Data augmentation generative adversarial networks," arXiv preprint arXiv:1711.04340.

Aparicio, C., Lang, N. P., and Rangert, B. (2006). "Validity and clinical significance of biomechanical testing of implant/bone interface," Clinical oral implants research 17, 2-7.

560 Chetlur, S., Woolley, C., Vandermersch, P., Cohen, J., Tran, J., Catanzaro, B., and Shelhamer, E. (2014). "cudnn: Efficient primitives for deep learning," arXiv preprint arXiv:1410.0759.

Costa, Y. M., Oliveira, L. S., and Silla Jr, C. N. (2017). "An evaluation of convolutional neural networks for music classification using spectrograms," Applied soft computing 52, 28-38.

Dorogoy, A., Haïat, G., Shemtov-Yona, K., and Rittel, D. (2020). "Modeling ultrasonic wave propagation in a dental implant-Bone system," Journal of the mechanical behavior of biomedical materials 103, 103547.

Dubory, A., Rosi, G., Tijou, A., Lomami, H. A., Flouzat-Lachaniette, C.-H., and Haïat, G. (2020). "A cadaveric validation of a method based on impact analysis to monitor the femoral stem insertion," Journal of the mechanical behavior of biomedical materials 103, 103535.

570 Fraulob, M., Vayron, R., Le Cann, S., Lecuelle, B., Hériveaux, Y., Albini Lomami, H., FlouzatLachaniette, C.-H., and Haïat, G. (2020). "Quantitative ultrasound assessment of the influence of roughness and healing time on osseointegration phenomena," Scientific reports In press.

Georgiou, A., and Cunningham, J. (2001). "Accurate diagnosis of hip prosthesis loosening using a vibrational technique," Clinical Biomechanics 16, 315-323.

Gill, A., and Shellock, F. G. (2012). "Assessment of MRI issues at 3-Tesla for metallic surgical implants: findings applied to 61 additional skin closure staples and vessel ligation clips," Journal of cardiovascular magnetic resonance : official journal of the Society for Cardiovascular Magnetic Resonance 14, 3.

580 Goossens, Q., Leuridan, S., Heny̌̌, P., Roosen, J., Pastrav, L., Mulier, M., Desmet, W., Denis, K., and Vander Sloten, J. (2017). "Development of an acoustic measurement protocol to monitor acetabular implant fixation in cementless total hip Arthroplasty: A preliminary study," Medical engineering \& physics 49, 28-38.

Haïat, G., Naili, S., Grimal, Q., Talmant, M., Desceliers, C., and Soize, C. (2009). "Influence of a gradient of material properties on ultrasonic wave propagation in cortical bone: Application to axial transmission," J. Acoust. Soc. Am. 125, 4043-4052.

Haiat, G., Wang, H.-L., and Brunski, J. (2014). "Effects of biomechanical properties of the boneimplant interface on dental implant stability: from in silico approaches to the patient's mouth," Annual review of biomedical engineering 16, 187-213.

590 Hériveaux, Y., Haïat, G., and Nguyen, V.-H. (2020). "Reflection of an ultrasonic wave on the bone-implant interface: Comparison of two-dimensional and three-dimensional numerical models," J. Acoust. Soc. Am. 147, EL32-EL36.

Hériveaux, Y., Nguyen, V.-H., Brailovski, V., Gorny, C., and Haiat, G. (2019a). "Reflection of an ultrasonic wave on the bone- implant interface: Effect of the roughness parameters," J. Acoust. Soc. Am. 145, 3370-3381.

Hériveaux, Y., Nguyen, V.-H., Geiger, D., and Haïat, G. (2019b). "elastography of the boneimplant interface," Scientific reports 9, 1-11. 
Hériveaux, Y., Nguyen, V.-H., and Haïat, G. (2018). "Reflection of an ultrasonic wave on the bone-implant interface: A numerical study of the effect of the multiscale roughness," $\mathrm{J}$.

600 Acoust. Soc. Am. 144, 488-499.

Hinton, G. E., Srivastava, N., Krizhevsky, A., Sutskever, I., and Salakhutdinov, R. R. (2012). "Improving neural networks by preventing co-adaptation of feature detectors," arXiv preprint arXiv:1207.0580.

Ioffe, S., and Szegedy, C. (2015). "Batch normalization: Accelerating deep network training by reducing internal covariate shift," arXiv preprint arXiv:1502.03167.

Khan, J., Wei, J. S., Ringner, M., Saal, L. H., Ladanyi, M., Westermann, F., Berthold, F., Schwab, M., Antonescu, C. R., and Peterson, C. (2001). "Classification and diagnostic prediction of cancers using gene expression profiling and artificial neural networks," Nature medicine 7, 673-679.

610 Khan, S. N., Ramachandran, M., Kumar, S. S., Krishnan, V., and Sundaram, R. (2012). "Osseointegration and more-A review of literature," Indian Journal of Dentistry 3, 72-76.

Kingma, D. P., and Ba, J. (2014). "Adam: A method for stochastic optimization," arXiv preprint arXiv:1412.6980.

Krizhevsky, A., Sutskever, I., and Hinton, G. E. (2012). "Imagenet classification with deep convolutional neural networks," in Advances in neural information processing systems, pp. 1097-1105.

Kwak, Y. (2018). "Acoustic Source Localization via Predicting Propagated Waves on Complex Systems using Time Reversal and Deep Neural Networks," Dissertation for the degree of doctor of philosophy, Hanyang University.

620 Kwak, Y., Kim, D., Ham, H., and Park, J. (2021). "Convolutional neural network trained with synthetic pseudo-images for detecting an acoustic source," Applied Acoustics 179, 108068.

Kwak, Y., Park, S. M., Lee, J., and Park, J. (2020). "Rattle noise source localization through the time reversal of dispersive vibration signals on a road vehicle," Wave Motion 93, 102452.

Mathieu, V., Fukui, K., Matsukawa, M., Kawabe, M., Vayron, R., Soffer, E., Anagnostou, F., and Haiat, G. (2011). "Micro-Brillouin scattering measurements in mature and newly formed bone tissue surrounding an implant," Journal of biomechanical engineering 133.

Mathieu, V., Vayron, R., Richard, G., Lambert, G., Naili, S., Meningaud, J.-P., and Haiat, G. (2014). "Biomechanical determinants of the stability of dental implants: Influence of the bone-implant interface properties," Journal of biomechanics 47, 3-13.

630 Mathieu, V., Vayron, R., Soffer, E., Anagnostou, F., and Haïat, G. (2012). "Influence of healing time on the ultrasonic response of the bone-implant interface," Ultrasound in medicine \& biology 38, 611-618.

Meredith, N., Alleyne, D., and Cawley, P. (1996). "Quantitative determination of the stability of the implant-tissue interface using resonance frequency analysis," Clinical oral implants 635 research 7, 261-267.

Michel, A., Bosc, R., Meningaud, J.-P., Hernigou, P., and Haiat, G. (2016). "Assessing the acetabular cup implant primary stability by impact analyses: a cadaveric study," PloS one 11.

Moerman, A., Zadpoor, A. A., Oostlander, A., Schoeman, M., Moshtagh, P. R., Pouran, B., and Valstar, E. (2016). "Structural and mechanical characterisation of the peri-prosthetic tissue surrounding loosened hip prostheses. An explorative study," Journal of the mechanical behavior of biomedical materials 62, 456-467. 
Nair, V., and Hinton, G. E. (2010). "Rectified linear units improve restricted boltzmann machines," in Proceedings of the 27th international conference on machine learning (ICML-10), pp. 807-814.

Njeh, C., Hans, D., Wu, C., Kantorovich, E., Sister, M., Fuerst, T., and Genant, H. (1999). "An in vitro investigation of the dependence on sample thickness of the speed of sound along the specimen," Medical engineering \& physics 21, 651-659.

Park, S. M., Visbal-Onufrak, M. A., Haque, M. M., Were, M. C., Naanyu, V., Hasan, M. K., and Kim, Y. L. (2020). "mHealth spectroscopy of blood hemoglobin with spectral superresolution," Optica 7, 563-573.

Pastrav, L. C., Jaecques, S. V., Jonkers, I., Van der Perre, G., and Mulier, M. (2009). "In vivo evaluation of a vibration analysis technique for the per-operative monitoring of the fixation of hip prostheses," Journal of orthopaedic surgery and research 4, 10.

655 Pattijn, V., Jaecques, S., De Smet, E., Muraru, L., Van Lierde, C., Van der Perre, G., Naert, I., and Vander Sloten, J. (2007). "Resonance frequency analysis of implants in the guinea pig model: influence of boundary conditions and orientation of the transducer," Medical engineering \& physics 29, 182-190.

Pattijn, V., Van Lierde, C., Van der Perre, G., Naert, I., and Vander Sloten, J. (2006). "The resonance frequencies and mode shapes of dental implants: Rigid body behaviour versus bending behaviour. A numerical approach," Journal of biomechanics 39, 939-947.

Perez, L., and Wang, J. (2017). "The effectiveness of data augmentation in image classification using deep learning," arXiv preprint arXiv:1712.04621.

Pilliar, R., Lee, J., and Maniatopoulos, C. (1986). "Observations on the effect of movement on bone ingrowth into porous-surfaced implants," Clinical orthopaedics and related research, $108-113$.

Salamon, J., and Bello, J. P. (2017). "Deep convolutional neural networks and data augmentation for environmental sound classification," IEEE Signal Processing Letters 24, 279-283.

Sasso, M., Haïat, G., Yamato, Y., Naili, S., and Matsukawa, M. (2007). "Frequency dependence of ultrasonic attenuation in bovine cortical bone: an in vitro study," Ultrasound in medicine \& biology 33, 1933-1942.

Sasso, M., Haïat, G., Yamato, Y., Naili, S., and Matsukawa, M. (2008). "Dependence of ultrasonic attenuation on bone mass and microstructure in bovine cortical bone," Journal of biomechanics 41, 347-355.

675 Schulte, W., and Lukas, D. (1992). "The Periotest method," International dental journal 42, 433440.

Seong, W.-J., Kim, U.-K., Swift, J. Q., Hodges, J. S., and Ko, C.-C. (2009). "Correlations between physical properties of jawbone and dental implant initial stability," The Journal of prosthetic dentistry 101, 306-318.

680 Shalabi, M., Wolke, J., Cuijpers, V., and A Jansen, J. (2007). Evaluation of bone response to titanium-coated polymethyl methacrylate resin (PMMA) implants by X-ray tomography.

Silver, D., Huang, A., Maddison, C. J., Guez, A., Sifre, L., Van Den Driessche, G., Schrittwieser, J., Antonoglou, I., Panneershelvam, V., and Lanctot, M. (2016). "Mastering the game of Go with deep neural networks and tree search," nature 529, 484.

685 Simonyan, K., and Zisserman, A. (2014). "Very deep convolutional networks for large-scale image recognition," arXiv preprint arXiv:1409.1556.

Strnad, G., and Chirila, N. (2015). "Corrosion rate of sand blasted and acid etched Ti6Al4V for dental implants," Procedia Technology 19, 909-915. 
Toh, G., and Park, J. (2020). "Review of Vibration-Based Structural Health Monitoring Using Deep Learning," Applied Sciences 10, 1680.

Van, D. S., and Wilson, C. (1991). "The Periotest method for determining implant success," The Journal of oral implantology 17, 410-413.

Vayron, R., Barthel, E., Mathieu, V., Soffer, E., Anagnostou, F., and Haiat, G. (2012). "Nanoindentation measurements of biomechanical properties in mature and newly formed bone tissue surrounding an implant," Journal of biomechanical engineering 134.

Vayron, R., Karasinski, P., Mathieu, V., Michel, A., Loriot, D., Richard, G., Lambert, G., and Haiat, G. (2013). "Variation of the ultrasonic response of a dental implant embedded in tricalcium silicate-based cement under cyclic loading," Journal of biomechanics 46, 11621168.

700 Vayron, R., Mathieu, V., Michel, A., and Haïat, G. (2014a). "Assessment of in vitro dental implant primary stability using an ultrasonic method," Ultrasound in medicine \& biology 40, 28852894.

Vayron, R., Matsukawa, M., Tsubota, R., Mathieu, V., Barthel, E., and Haiat, G. (2014b). "Evolution of bone biomechanical properties at the micrometer scale around titanium implant as a function of healing time," Physics in Medicine \& Biology 59, 1389.

Vayron, R., Nguyen, V.-H., Bosc, R., Naili, S., and Haiat, G. (2016). "Assessment of the biomechanical stability of a dental implant with quantitative ultrasound: A threedimensional finite element study," J. Acoust. Soc. Am. 139, 773-780.

Vayron, R., Nguyen, V.-H., Bosc, R., Naili, S., and Haïat, G. (2015). "Finite element simulation of ultrasonic wave propagation in a dental implant for biomechanical stability assessment," Biomechanics and modeling in mechanobiology 14, 1021-1032.

Vayron, R., Nguyen, V.-H., Lecuelle, B., Albini Lomami, H., Meningaud, J.-P., Bosc, R., and Haiat, G. (2018a). "Comparison of resonance frequency analysis and of quantitative ultrasound to assess dental implant osseointegration," Sensors 18, 1397.

715 Vayron, R., Nguyen, V. H., Lecuelle, B., and Haiat, G. (2018b). "Evaluation of dental implant stability in bone phantoms: Comparison between a quantitative ultrasound technique and resonance frequency analysis," Clinical implant dentistry and related research 20, 470-478.

Vayron, R., Soffer, E., Anagnostou, F., and Haïat, G. (2014c). "Ultrasonic evaluation of dental implant osseointegration," Journal of biomechanics 47, 3562-3568.

Vesperini, F., Vecchiotti, P., Principi, E., Squartini, S., and Piazza, F. (2018). "Localizing speakers in multiple rooms by using Deep Neural Networks," Comput. Speech Lang. 49, 83-106.

Yosinski, J., Clune, J., Nguyen, A., Fuchs, T., and Lipson, H. (2015). "Understanding neural networks through deep visualization," arXiv preprint arXiv:1506.06579.

\section{Table}

Table I. Material properties used in the numerical simulations. Values taken from (Njeh et al., 1999; Pattijn et al., 2006; Pattijn et al., 2007; Haïat et al., 2009). 


\begin{tabular}{lccc}
\hline \hline & $C_{p}\left(\mathrm{~m} \cdot \mathrm{s}^{-1}\right)$ & $C_{s}\left(\mathrm{~m} \cdot \mathrm{s}^{-1}\right)$ & $\rho\left(\mathrm{kg} \cdot \mathrm{m}^{-3}\right)$ \\
\hline Soft tissue (liquid) & 1500 & 10 & 1000 \\
Titanium & 5810 & 3115 & 4420 \\
Cortical bone tissue & 4000 & 1800 & 1850 \\
\hline \hline
\end{tabular}

\section{Figure Legends}

740 FIG 1. (a) Schematic illustration of the geometrical configuration of the BII considered in the numerical simulation. (b) Description of the streamlined model.

FIG 2. Layer structure of the proposed CNN to assess the soft tissue thickness $W$. $W^{e}$ corresponds 745 to the estimated soft tissue thickness.

FIG. 3. Radiofrequency signals corresponding to the ultrasound responses of the bone-implant interface for $h=360 \mu \mathrm{m}, L=900 \mu \mathrm{m}$ (macroscopic case) and $W / h=$ (a) 0 , (b) 0.5 , (c) 1 . The solid grey lines indicate the results obtained using the proposed model (in the frequency domain) and

750 the dashed black lines indicate the results obtained in (Hériveaux et al., 2018) (in the time domain).

FIG. 4. Variation of the reflection coefficient $R$ for different values of the roughness amplitude $h$ as function of (a) the soft tissue thickness $W$ in the microscopic case $(L=50 \mu \mathrm{m})$ and (b) the ratio of the soft tissue thickness $W$ and of the roughness amplitude $h$ in the macroscopic case ( $L=900$ $755 \mu \mathrm{m})$. The symbols indicate the result obtained by the proposed model in the frequency domain for the different values of $h$, and the dashed lines indicate the corresponding result obtained using the time-domain model in (Hériveaux et al., 2018). 
FIG. 5. Spectrograms of the ultrasonic signals as a function of the soft tissue thickness. (a)-(d) For

760 the microscopic case, $h=5 \mu \mathrm{m}, L=50 \mu \mathrm{m}$ and (a): $W=0 \mu \mathrm{m}$, (b): $25 \mu \mathrm{m}$, (c): $50 \mu \mathrm{m}$, (d): 100 $\mu \mathrm{m}$. (e)-(h) For the macroscopic case, $h=360 \mu \mathrm{m}, L=900 \mu \mathrm{m}$ and (e): $W / h=0$, (f): 0.5, (g): 1.0, (h): 1.5 .

FIG. 6. Representative feature maps of the convolution layers for the reference BII roughness 765 cases, separated from the training dataset. (a)-(d) For $h=5 \mu \mathrm{m}, L=50 \mu \mathrm{m}$ for the microscopic roughness, the results from the first and the second convolution layers for the case of $W=50 \mu \mathrm{m}$ ((a)\&(c), respectively) and $100 \mu \mathrm{m}$ ((b)\&(d), respectively). (e)-(h) For $h=360 \mu \mathrm{m}, L=900 \mu \mathrm{m}$ for the macroscopic roughness, the results from the first and the second convolution layers for the case of $W / h=0.5((\mathrm{e}) \&(\mathrm{~g})$, respectively) and $1.0((\mathrm{f}) \&(\mathrm{~h})$, respectively).

FIG. 7. Comparison between the actual and estimated values of the soft tissue thickness using the proposed convolutional neural network applied to the ultrasound reflections on the BII for (a) $h=$ $5 \mu \mathrm{m}, L=50 \mu \mathrm{m}$ (the microscopic roughness), (b) and $h=360 \mu \mathrm{m}, L=900 \mu \mathrm{m}$ (the macroscopic 775 roughness). 